II. 


\section{Unwissen, Gerücht, Literatur \\ Der Giftmord in den Zeiten vor der Marshschen Probe}

I.

Es ist nicht schwer zu verstehen, weshalb der Giftmord die einzige Tötungsart ist, die auch nach dem Tötungsmittel benannt ist. ${ }^{1}$ Das Tötungsmittel, das Medium, versetzt den Täter beim Giftmord in eine besondere Subjektposition. Er muss nämlich nicht als Täter in Erscheinung treten; er setzt nur eine Kausalkette in Gang. Die Tat wird als solche nicht sichtbar. Es gibt bei diesem gewaltsamen Tod keine Szene der Gewalt. Die Tat selbst entzieht sich der Darstellung. Das Opfer stirbt gleichsam von selbst. Man könnte sagen: Beim Giftmord spielt der Täter Schicksal für sein Opfer: Das Opfer stirbt einen Tod, der von einem natürlichen - schicksalhaften - Tod durch Krankheit von aufsen nicht zu unterscheiden ist. Die Tat ahmt die Natur nach. ${ }^{2}$ Dass man den Giftmord nicht sehen kann, hat zur Folge, dass er zu einem Phantasma wird. Insofern er nicht nachweisbar ist, lässt sich der Giftmord nicht lokalisieren. Vom Augenschein her kann man nicht wissen, ob bei entsprechenden Symptomen eine Tat vorliegt oder nicht. Um die Tat nachzuweisen, müsste man im Prinzip eine Naturwissenschaft betreiben - Chemie: Nur sie könnte das Gift im Körper des Toten nachweisen. Außerhalb dieses Nachweises gibt es nur den Verdacht. Und der Verdacht kann sich ebenso einstellen, wo keine Tat vorliegt. Daher rührt dann die krankhafte, paranoische Angst, vergiftet zu werden. ${ }^{3}$

1 Vgl. Liselotte Herx: Der Giftmord, insbesondere der Giftmord durch Frauen, Emsdetten 1937, S. 12.

2 Ich habe dies an anderer Stelle ausführlich als `Giftmord-Komplex` beschrieben; vgl. Michael Niehaus: Die Figur der Giftmischerin als Fall der Literatur, in: KulturPoetik 5/2 (2005), S. 153-168, insbes. S. 153-158.

3 In die Angst gehen beide Besonderheiten des Giftmordes ein: erstens die Unsichtbarkeit der Tat bzw. das Fehlen der Szene der Tat (ich weiß nicht, ob ich, indem ich dies zu mir nehme, meiner eigenen Vergiftung beiwohne bzw. sie bewirke), und zweitens die Unnachweisbarkeit der Tat (ich weiß nicht, ob Menschen aus meinem Umkreis, die vor mir plötzlich gestorben oder erkrankt sind, vergiftet worden sind). Diese beiden Ängste verzahnen sich. 
Es ist folglich kein Zufall, dass der Giftmord schon in der römischen Antike eine Sonderstellung einnimmt. Auch damals konnte man kaum sagen, wie häufig das Vorkommen dieses Deliktes war, weil es möglicherweise sehr viel häufiger gemutmaßt als wirklich ausgeführt wurde; jedenfalls war die Angst vor Vergiftung weit verbreitet, ${ }^{4}$ während die Aufklärungsrate mangels Nachweismöglichkeit gering blieb (gleichwohl wurde im ersten Jahrhundert vor Christus unter Sulla ein Gerichtshof eingerichtet, der sich eigens mit Bandenkriminalität und Giftmord beschäftigte). ${ }^{5}$

Schon in der römischen Antike übrigens wird dieses Verbrechen vor allem den Frauen zugeordnet: Die Sphäre der Tatausführung ist naheliegender Weise das Haus, der häusliche Herd, wo vorzugsweise diejenigen zu diesem Mittel greifen, die nicht die potestas innehaben, die nicht das Oberhaupt sind (aber in einem nahen Verhältnis zu ihm stehen, indem sie ihm etwa die Speisen reichen). Eine besondere Blütezeit (wenn man so sagen darf) erlebte der Giftmord im Italien der Renaissance und im absolutistischen Frankreich. Der Gebrauch des Giftes als Tötungsmittel blieb dabei weitgehend den adligen Schichten und der hohen Geistlichkeit vorbehalten. Man kann den Giftmord auf einer strukturellen Ebene als genealogisches Verbrechen betrachten, ${ }^{6}$ da es häufig die Verwandten im eigenen Hause betrifft und dazu dient, in die Erbfolge einzugreifen oder sie zu beschleunigen. Er besitzt also eine Nähe zum Verwandtenmord, zum Parrizid. Im Frankreich Ludwigs XIV. trug das beliebte Arsen bzw. Arsenik den Beinamen poudre de succession - "Erbschaftspulver ${ }^{7}$

Solange sich das Gift im Körper nicht nachweisen lässt, sind die Ermittlungsbemühungen - abgesehen von der Unnachweisbarkeit des Verbrechens - in einem weiteren entscheidenden Nachteil. Weil die Tat keine Szene ist, kann der Täter auch durch Tatzeugen kaum überführt werden. Gewiss kann man hoffen, bei verdächtigen Todesfällen Giftküchen zu finden, wie im Falle der berühmten Marquise des Brinvillier, der ersten neuzeitlichen Heroine des Giftmords, deren Untaten während der Herrschaft unter Ludwig XIV. von

4 Vgl. Jens-Uwe Krause: Kriminalgeschichte der Antike, München 2004, S. 132ff. Zur Verbreitung und Nachweisbarkeit von Giften allgemein vgl. das voluminöse Werk von Louis Lewin: Die Gifte in der Weltgeschichte, Berlin 1920, zu den Vergiftungen im antiken Rom S. $184 \mathrm{ff}$.

5 Vgl. Jens-Uwe Krause: Kriminalgeschichte der Antike, S. 132.

6 Den Begriff des genealogischen Verbrechens entleihe ich dem Juristen und Psychoanalytiker Pierre Legendre (vgl. etwa Pierre Legendre: Das Verbrechen des Gefreiten Lortie, Freiburg i.Br. 1998, S. 105ff.).

7 Liselotte Herx: Der Giftmord, S. 7. Die Bezeichnung findet auch in E. T. A. Hoffmanns Fräulein von Scuderi Verwendung. 
Guyot de Pitaval Mitte des 18. Jahrhunderts in seiner berühmten Fallsammlung aufbereitet wurden. ${ }^{8}$ Aber eine Giftküche ist kein Tatbeweis. Sicher kann es auch geschehen, dass die vergiftete Speise auffällt und abgefangen wird, die Vergiftung also in flagranti bewiesen werden kann - aber dann ist die Tat freilich noch nicht vollendet, und der Täter kann immerhin noch leugnen, es auf den Tod des Betreffenden abgesehen zu haben (und diese Behauptung hat insofern etwas für sich, als die Zahl der bloßen Vergiftungsversuche ohne unmittelbare Todesfolge sehr hoch ist).

Da die Praktiken der Ermittlung beim bereits verübten Verbrechen wenig aussichtsreich sind, hat man dessen Begehung vor allem im Vorfeld zu verhindern gesucht: Der Besitz von Gift wird unter Strafe gestellt, seine Abgabe streng kontrolliert usw. Das galt besonders für Arsenik - Arsentrioxid. Dies war seit der Spätantike das am einfachsten zu beschaffende Gift. Im neuzeitlichen Europa wurde es von Apotheken als Rattengift oder Mäusebutter vertrieben. Besonderer Beliebtheit erfreute es sich aber auch, weil es die Natur besonders gut nachahmte: In entsprechenden Dosen verabreicht, führte es nicht zu einem plötzlichen Tode und die hauptsächlichen Symptome - Erbrechen, Fieber, Schwäche, Krämpfe - konnten sehr gut auch auf andere Krankheiten zurückgeführt werden.

Das änderte sich 1836. In diesem Jahr veröffentlichte der britische Chemiker James Marsh (1794-1846) eine Methode, mit der Arsentrioxid noch in außerordentlich geringen Mengen von bis zu 0,1 Mikrogramm in Leichen nachgewiesen werden konnte. ${ }^{9}$ Zwar hatte es bereits Vorläufer solcher Nachweisverfahren in Deutschland gegeben, aber erst mit diesem Verfahren konnte der Nachweis einwandfrei erbracht werden. ${ }^{10}$ Seitdem ist die Zahl der Giftmorde deutlich zurückgegangen. Marsh selbst schließt seine

8 Gayott von Pitaval: Erzählung sonderbarer Rechtshändel, sammt deren gerichtlichen Entscheidung. Aus dem französischen übersetzt. Erster Theil, Leipzig 1747, S. 331-390.

9 Ein Jahr später auch in deutscher Übersetzung: James Marsh: Beschreibung eines neuen Verfahrens, um kleine Quantitäten Arsenik von den Substanzen abzuscheiden, womit er gemischt ist, in: Annalen der Pharmacie, Bd. 23/2 (1837), S. 207-216.

10 Die Zeichen, die an einem bereits seit längerem toten Körper zu finden sind, gelten noch bis Ende des 19. Jahrhunderts als weitgehend unlesbar bzw. extrem unsicher, da sie von den Zeichen der Verwesung gewissermaßen überschrieben sind. Stephanie Langer stellt fest: "Die gerichtliche Arzneikunde der Zeit [Ende des 18. Jahrhunderts, M.N.] ringt um ein klares Begriffsinstrumentarium, stößt dabei jedoch im Zusammenhang mit Vorgängen der Verwesung auf erhebliche Schwierigkeiten. Die Verwesung markiert nämlich den toten Körper als dem Zugriff des medizinischen Blicke entzogen." Stephanie Langer: Gedächtnis post mortem. Der tote Körper als Archiv, in: Naturgeschichte, Körpergedächtnis. Erkundungen einer kulturanthropologischen Denkfigur, hg. von Andrea Bartl und Hans-Joachim Schott, Würzburg 2014, S. 371-388, hier S. 376. 
Ausführungen gar mit den Worten: "Man sollte glauben, daß wenn die Kenntniß dieser empfindlichen und untrüglichen Probe auf Arsenik allgemein bekannt wäre, gar kein Versuch einer heimlichen Vergiftung mehr gemacht würde. «11

Dies müsste insbesondere auf wiederholte Vergiftungstaten zutreffen. 1842 - also wenige Jahre später - haben Julius Eduard Hitzig und Wilhelm Häring im zweiten Band ihres Neuen Pitaval, der prägenden Sammlung von Kriminalrechtsfällen im 19. Jahrhundert, ${ }^{12}$ vier Wiederholungstäterinnen, vier Giftmischerinnen, ausführlich porträtiert (wobei in unterschiedlichem Umfang auf bereits vorliegende Falldarstellungen zurückgegriffen wurde). In der Folge wurde dieses »Viergespann der Giftmischerinnen « ${ }^{13}$ immer wieder in einem Atemzug genannt und hat trotz der Verschiedenartigkeit der vier Täterinnen das Bild der weiblichen Giftmischerin entscheidend geprägt. ${ }^{14}$ Es handelt sich um die Marquise de Brinvillier (1676), die verwitwete Geheimrätin Charlotte Ursinus (1803), die Dienstbotin Anna Margaretha Zwanziger (1811) und die Bremer Bürgerin Gesche Gottfried geborene Timm (1831). Mit zweien von ihnen - Charlotte Ursinus und Gesche Gottfried - möchte ich mich nun hinsichtlich des Verhältnisses von Verbrechen und Ermittlung ein wenig beschäftigen. Die beiden Fälle sollen etwas durchaus Allgemeines exemplifizieren, das freilich in einer besonderen historischen Situation - nämlich der am Horizont stehenden Nachweisbarkeit des Giftes - hervortritt.

II.

Der Fall der Charlotte Ursinus ist alles andere als kompliziert. Die 1760 geborene Tochter eines des Hochverrats bezichtigten und daher nach Preußen ausgewanderten österreichischen Barons hatte im Alter von 19 Jahren einen Freund des elterlichen Hauses, den eine Generation älteren und

11 James Marsh: Beschreibung eines neuen Verfahrens, S. 216.

12 Vgl. dazu ausführlich den Beitrag von Carsten Zelle in diesem Band.

13 Der Neue Pitaval. Eine Sammlung der interessantesten Criminalgeschichten aller Länder aus älterer und neuerer Zeit. Zweiter Theil, hg. von Julius Eduard Hitzig und Wilhelm Häring, Leipzig 1842, S. 253. Im Folgenden die Nachweise im Text mit der Sigle NP.

14 Vgl. dazu ausführlich Inge Weiler: Giftmordwissen und Giftmörderinnen. Eine diskursgeschichtliche Studie, Tübingen 1998. Weiler steht sich mit ihrer Vorannahme, dass der Neue Pitaval das Bild der weiblichen Giftmischerin tatsächlich blo/s konstruiere, selbst im Weg. 
kränklichen Obergerichtsrat Ursinus geheiratet. 1792 zog das Paar nach Berlin, wo Charlotte Ursinus bald ein angesehenes Mitglied der vornehmen Kreise wurde. Das änderte sich auch nach dem Tod ihres Mannes im Jahre 1800 nicht.

Die großartige Falldarstellung im Neuen Pitaval beginnt mit der Szene der Enttarnung von Charlotte Ursinus am 5. März 1803, die - so Wilhelm Häring noch fast 40 Jahre später »in Vieler Gedächtniß« lebe, weil sie ein »Ereigniß so außergewöhnlicher Art " gewesen sei, »daß es den ruhigen Lebensstrom des friedlichen Berlins jener Tage völlig unterbrach" (NP 161). 'Die Ursinus wird am Spieltisch verhaftet. Sie legt scheinbar ungerührt die Whistkarten weg und bittet ihre Mitspieler wegen der Unterbrechung um Entschuldigung. Der faszinierte Wilhelm Häring hat diese Szene zehn Jahre später in dem unter seinem Pseudonym Willibald Alexis erschienenen historischen Roman Ruhe ist die erste Bürgerpflicht noch einmal gestaltet. ${ }^{15}$ Die Geheimrätin heißt dort Lupinus statt Ursinus und steht exemplarisch für den Zustand Preußens vor der großen Niederlage gegen Napoleon.

Exemplarisch ist die Szene auch hinsichtlich des Verhältnisses von Tötungsart und Ermittlung. Der bzw. die Tatverdächtige wird bei der Giftmordanklage strukturell aus der Mitte der Gesellschaft gerissen, weil die Tat selbst, bevor die Verhaftung erfolgt, als solche noch gar nicht bekannt und aktenkundig ist. Der Bezichtigte ist nicht ein bereits gesuchter Täter, vielmehr wird das Vorliegen einer Tat erst dadurch statuiert, dass jemand einer Tat bexichtigt wird. Die Verhaftung steht nicht am Ende der Ermittlung, sondern erst in der Verhaftung wird offenbar, dass es überhaupt eine Ermittlung gibt. Überspitzt formuliert, wird die Welt im Augenblick der 'Enttarnung` in ein anderes Licht getaucht.

In diesem Fall wurde schnell ruchbar, dass Charlotte Ursinus ihrem Bedienten und Vertrauten namens Benjamin Klein verschiedenen Nahrungsmitteln beigemengtes Arsenik in kleineren Dosen hatte zukommen lassen, bis dieser mit Arsenik verseuchte Pflaumen zu einem Apotheker brachte und analysieren ließ. Insoweit handelte es sich also um die Variante des manifesten Vergiftungsversuches. Im Neuen Pitaval heißt es aber weiter:

Dies wusste man in den ersten Stunden. Bald wusste man weit mehr. Auch ihr Gatte war vor drei Jahren an Gift gestorben, das sie ihm beigebracht, desgleichen ihre

15 Vgl. Willibald Alexis: Ruhe ist die erste Bürgerpflicht, Frankfurt a.M./Berlin/Wien 1985, Bd. 2, S. 358f. Zur Einordnung von Wilhelm Häring alias Willibald Alexis vgl. Wolfgang Beutin/Peter Stein (Hg.): Willibald Alexis (1798-1871). Ein Autor des Vor- und Nachmärz, Bielefeld 2000. 
Tante, die unverehelichte Witte, und ebenso ein Geliebter der Ursinus, ein holländischer Offizier, Namens Ragay. (NP 163)

Ein Unwissen wird hier ironisch als Wissen deklariert, um das Gerücht zu bezeichnen. Im weiteren Verlauf des Verfahrens wird sich herausstellen, dass es keinerlei Verdachtzeichen für die Ermordung des Geliebten gibt und dass Charlotte Ursinus nach Exhumierung die Vergiftung ihres Gatten nicht nachgewiesen werden kann. ${ }^{16} \mathrm{Zu}$ lebenslänglicher Haft wird sie wegen der erwiesenen Vergiftungsversuche an ihrem Bedienten und der mutma/slichen Vergiftung ihrer Tante verurteilt. Dass die erste Tat nicht vollendet wurde, bei der anderen aber letzte Zweifel bleiben, rettet sie nach dem in Preußen geltenden Inquisitionsverfahren vor der Verhängung der gesetzlichen Todesstrafe - während sie, wie der Neue Pitaval notiert, "vor einem Geschworenengerichte ein unbedingtes Schuldig« (NP 179) zu gewärtigen gehabt hätte. ${ }^{17}$

Aber auch das vermeintliche Wissen um die weiteren Taten der Ursinus genügte dem "Publicum in Berlin", wie der Neue Pitaval ausführt, nicht. Dieses war »entbrannt darauf, noch mehr zu wissen«. Denn ist die Öffentlichkeit einmal in einen erregten Zustand versetzt, dann ist sie bereit zu glauben und verlangt, »unersättlich, nach dem Unglaublichen.« (NP 163) Diese Affinität zum Phantasmatischen, die dem enthüllten Giftmord eignet, muss von der Ermittlung zurückgewiesen werden. Die Enthüllung fungiert als ein auslösendes Moment, das ein ganzes Konglomerat von Vorstellungen freisetzt. Dabei geht es nicht nur um mögliche weitere Taten, sondern auch um die Bearbeitung von mit dem Giftmord zusammenhängenden Fragen und um die Möglichkeitsbedingung solcher Taten: Auf welchem Boden gedeihen sie? Inwiefern sind sie exemplarisch? An der Figur der Giftmischerin kondensieren sich fundamentale Fragen der abendländischen Zivilisation; sie

16 Valentin Rose der Jüngere (1762-1807) entwickelte nach seinen Erfahrungen mit dem gescheiterten Nachweisverfahren im Fall Ursinus ein erstes, noch nicht 100-prozentiges Verfahren für den Nachweis von Arsenik; vgl. Valentin Rose: Über das zweckmäßige Verfahren, um bei Vergiftungen mit Arsenik letzteren aufzufinden und darzustellen, in: Gehlens Jahrbuch für Chemie, Physik und Mineralogie 2 (1806), S. 665-671.

17 In weiten Teilen Deutschlands trat in der napoleonischen Zeit das mündlich-öffentliche Gerichtsverfahren an die Stelle des schriftlichen und nichtöffentlichen Inquisitionsverfahrens; seit dieser Zeit herrschte eine teils erbitterte Auseinandersetzung um die Vorzüge der beiden Verfahren; Peter Friedrich/Michael Niehaus: Transparenz und Maskerade. Zur Diskussion über das mündlich-öffentliche Gerichtsverfahren um 1800 in Deutschland, in: Poetologien des Wissens um 1800, hg. von Joseph Vogl, München 1999, S. 163-185. 
lässt in einen Abgrund schauen. ${ }^{18}$ Erst die ordnungsgemäße Beendigung des Verfahrens erlaubt es, solche Fragen wieder ad acta zu legen. Aber dazu braucht es Zeit. In der Zwischenzeit kann die Literatur dem Begehren, in einen Abgrund zu schauen, eine Antwort und einen Resonanzboden geben. Das hat sie in diesem Fall getan. Es verwundert freilich nicht, dass diese Antwort Hehlerware ist. Noch im Jahr der Verhaftung erschien - anonym - das Buch Bekenntnisse einer Giftmischerin. Von ihr selbst geschrieben, welches - so der Neue Pitaval - »[v]ielen für baare Münze galt; doch aber nur ein Roman ist, dessen Hauptmotive allein Dem entnommen sind, was vor der Publication des Erkenntnisses der Untersuchung bekannt geworden war.« (NP 163f.) Mit dieser Einschätzung wird Häring dem Buch nicht gerecht. Der Autor dieses Buches war dem Gerücht nach Friedrich Buchholz, ein bekannter Berliner Publizist und Historiker. ${ }^{19}$ Eingangs erklärt der anonyme "Herausgeber" in einer kurzen Erklärung "An den Leser«, die »Aechtheit einer Schrift zu erweisen«, sei ein müßiges Bemühen: »der Unglaube steigt, je mehr man den Glauben erzwingen will«. Daher möchte er sich mit der folgenden Leseanweisung aus der Affäre ziehen: "Alles, was in diesen Bekenntnissen faktisch ist, oder als faktisch erscheint, so zu nehmen, daß dadurch ein merkwürdiges psychologisches Problem gelöset wird «. ${ }^{20}$

Das merkwürdige psychologische Problem besteht darin, eine ungeheuerliche Verbrecherin zu erklären, ohne dabei auf etwaige Naturanlagen zurückzugreifen. Entsprechend behauptet die anonym bleibende Giftmischerin in ihren Bekenntnissen von sich selbst, dass sie »von Natur gewiß ebenso we-

18 Vgl. Michael Niehaus: Schicksal sein. Giftmischerinnen in Falldarstellungen vom Pitaval bis zum Neuen Pitaval, in: Internationales Archiv für Sozialgeschichte der deutschen Literatur 31/1 (2006), S. 133-149.

19 Paul Ferdinand Friedrich Buchholz (1768-1843) lebte seit 1800 als Schriftsteller in Berlin. Bereits vor 1800 war er Mitarbeiter der führenden politischen Zeitschriften der Epoche. U.a. edierte er das Historische Taschenbuch oder Geschichte der europäischen Staaten seit dem Frieden von Wien (Berlin 1814-37, 22 Bde.). Nach 1815 verwandelte er sich aus einem Parteigänger Napoleons "in einen den deutschen Interessen eher aufgeschlossenen Publizisten" und leitete das fournal von und für Deutschland (Berlin 1815-19), das 1820 in Neue Monatsschrift fuir Deutschland (1820-35, 40 Bde.) umbenannt wurde. Buchholz galt als "glänzender Publizist« aber auch als »eitel, gewinnsüchtig und sogar bestechlich" (Wilmont Haacke: 'Buchholz, Paul Ferdinand Friedrich`, in: Neue Deutsche Biographie 2 (1955), S. 701f., http://www.deutsche-biographie.de/pnd118516663.html [Stand: 30.09.2015]).

20 [Paul Ferdinand Friedrich Buchholz]: Bekenntnisse einer Giftmischerin. Von ihr selbst geschrieben, Berlin 1803, S. 2f. Im Folgenden mit der Sigle BG ausgewiesen. Das Werk ist 1988 in der Reihe Die Frau in der Literatur bei Ullstein erneut aufgelegt worden, allerdings mit erheblichen, nicht kenntlich gemachten Kürzungen. Auch dort wird im Nachwort von Frank Dietschreit ausgeführt, dass das Buch einen großen Erfolg hatte und schnell zum Stadtgespräch wurde. 
nig bösartig war, als irgend ein Mensch« (BG 17). Die autobiographische Schrift muss also das Werden einer vollendeten Serienmörderin als »eine eng zusammenhängende Kette von Ursachen und Wirkungen" (BG 219) erweisen, an deren Ende diese über sich sagen kann: »Ich bin schlechterdings über alle Strafe hinaus, weil das, was ich gethan habe, nach meiner ganzen Individualität und den mitwirkenden Ursachen immer nothwendig war«. (BG 219).

Mit der historischen Charlotte Ursinus hat diese Giftmischerin recht wenig zu tun. Ihre Karriere beginnt, als sie zur Mitwisserin wird, wie der geliebte Vater seine todkranke Frau vergiftet und die eigene Tochter in der Folge, um sie an sich zu binden, zu seiner "Beischläferin« (BG 51) macht. Im Alter von 18 Jahren und mit einiger Erfahrung in Liebesdingen wähnt sich die künftige Giftmischerin so reif wie andere Frauen doppelten Alters. Nach ihrer Heirat verzweifelt sie zunächst daran, offenbar keine Kinder bekommen zu können. All dies wird mit viel Einfühlungsvermögen geschildert; so auch der plötzliche Entschluss zur entscheidenden ersten Tat - dem Mord am ungeliebten und kränklichen Ehemann um eines sich entziehenden Liebhabers willen -, nachdem ihr beim Durchsuchen des väterlichen Schreibtisches unvermutet das Gift in die Hände gefallen ist, mit dem dieser das Leben seiner Frau verkürzt hatte. Die Selbstrechtfertigung der Tat erfolgt nicht zufällig unter Rückgriff auf das Geschlechterverhältnis:

Was bleibt, sagte ich zu mir selbst, deinem Geschlechte anders übrig als die List, da Natur und Gesellschaft sich verschworen haben, ihm die Gewalt zu nehmen? Was für einen Mann ein Verbrechen seyn würde, ist keins für ein Weib. Die Gesetze - was sind sie anders, als Tyrannen, denen man sich entziehen muß? Und ist es deine Schuld, dass du genöthigt bist, deine Freiheit durch solche Mittel zu erkaufen? (BG 156)

Die Zwiespältigkeit dieser männlichen Einfühlung in die Lage der Frau wird hier manifest. Nicht nur affirmiert sie den Giftmord als ein weibliches Verbrechen, auch ist sie eine Projektion, aus der die Angst vor einer Selbstlegitimation des anderen Geschlechts zu einem Tätlichwerden spricht, dessen man nicht Herr werden kann.

Gleichwohl ist die Täterin noch an dieser Stelle insofern seine von uns`, als sie nach der Giftbeimischung zurückschreckt und ihr »halbes Leben aufgeopfert haben" würde, wenn sie ihn "hätte dadurch retten können« (BG 163). Freilich führt das anschließende Unentdecktbleiben dieser ersten Tat dazu, dass bereits der Mord an der Tante »mit der größten Kaltblütigkeit« (BG 192) ausgeführt wird. Weitere Verbrechen folgen - Morde, aber auch 
Diebstähle. Und es zeigt sich, dass die Bekenntnisse selbst - in der Fiktion sind sie als nach dem Tode der Schreiberin zu öffnender Brief an eine andere Frau konzipiert - mit keiner Reue verknüpft sind. Sie stellen vielmehr jemanden vor, der aus der sozialen Welt herausgefallen ist, der alle Bindungen gekappt hat, aber gleichzeitig unerkannt, als vollkommene Heuchlerin, in unserer Mitte lebt. Der männliche Verfasser dieses Buches spricht durch den Mund (bzw. die Feder) einer Frau, die einerseits vor dem Abgrund steht, aber andererseits ihr Leben in gewisser Weise auch als Nachrichten aus dem Abgrund erzählt:

Daß ich eine Verbrecherin bin, weiß ich; daß ich Verbrechen auf Verbrechen häufen muß, weiß ich nicht minder. Für mich findet keine Reue statt; noch weit weniger ein Umkehren von dem einmal betretenen Pfade [...]. Mit der übrigen Welt führ' ich den einmal angefangenen Kampf fort [...]. Unter jedem meiner Fußtritte eröffnet sich ein Abgrund. (BG 7f.)

Mit welchen Ermittlungspraktiken kann man einer solchen Frau beikommen? Das ist eine Frage, die Friedrich Buchholz natürlich nur am Rande interessiert, da für ihn das psychologische Rätsel im Vordergrund steht. Es ist aber eine Folge der Rätselfigur Giftmischerin, dass die Gesellschaft ihr letztlich nichts und insbesondere keine Ermittlungspraktiken entgegenzusetzen hat, da die Gesellschaft überhaupt nicht weiß, dass es etwas zu ermitteln gibt. Dass wir einer (weiblichen) Figur ohnmächtig gegenüberstehen, die einen Kampf und einen Krieg gegen uns führt, ohne dass wir es wissen, darin besteht ja das herbeizitierte (männliche) Phantasma.

Auf den letzten Seiten ihrer Bekenntnisse gibt Friedrich Buchholz' gescheite Giftmischerin ein paar Tipps, wie man Figuren ihren Schlages beikommen könnte. Allein das Bündnis von Polizei und Wissenschaft wäre dazu in der Lage - also die verbesserte Kriminalistik:

Wie hätte ich so ungescheut vergiften können, wenn es eine öffentliche Todtenschau gäbe [...], welche von sachkundigen Männern angestellt würde, deren Hauptgeschäft kein anderes wäre, als sich der natürlichen oder unnatürlichen Todesart der Ausgestellten zu versichern«? (BG 222)

Das Unentdecktbleiben des Verbrechens ist zugleich schon die halbe Lösung für das psychologische Rätsel der Figur der Giftmischerin. Denn unsere Giftmischerin wird nicht müde zu betonen, dass man durch »die Natur" eines solchen "Verbrechens zur Wiederholung desselben genöthigt werde« (BG 223). Die Giftmischerin verdient ihren Namen erst als Widerholungstäterin; das Unentdecktbleiben ihrer Tat reizt unfehlbar zu ihrer Wieder- 
holung an, die sie schließlich inmitten unserer Gesellschaft ins Jenseits der Gesellschaft führen muss. So sei die Brinvillier - das immer wieder zitierte Modell - dazu gekommen, »in einem Zeitraum von ungefähr zehn Jahren hundert und fünfzig Menschen« (BG 223) zu vergiften.

Deshalb gilt es den Anfängen zu wehren. Vorbeugende Polizeimaßnahmen inklusive Erziehung und wissenschaftlicher Fortschritt werden aber noch aus einem anderen Grunde als das einzige probate Mittel gegen die Giftmischerin vorgestellt. So, wie sie von Friedrich Buchholz konstruiert wird, ist der Giftmischerin mit dem Inquisitionsverfahren nicht beizukommen. In ihm ist die Verhängung der gesetzmäßigen Strafe nur bei Geständnis bzw. Überführung durch zwei Zeugen möglich. Die Bekenntnisse einer Giftmischerin machen aber deutlich, dass ein wahrhaftiges, rückhaltloses Geständnis von jemandem, der sich durch seine Taten im Jenseits der Gesellschaft situiert, nicht zu erwarten ist. Mit der Giftmischerin gäbe es, würde sie gefasst und wüsste sie sich nicht mehr zu entziehen, nur fruchtlose Verhöre - zumal nach Abschaffung der Folter. Denn das Verhör muss fruchtlos bleiben, insofern es nicht als der Beginn einer Rückkehr in die Kommunikationsgemeinschaft interpretiert werden kann. ${ }^{21}$

Wie verhält sich nun die Ursinus der Falldarstellung zu dieser literarischen Konstruktion der Rätselfigur Giftmischerin? Zwar wurde - so der Neue Pitaval - die »Erwartung, in der berliner Geheimräthin eine Brinvillier zu entdecken« (NP 164), enttäuscht, als Widerholungstäterin wurde sie gleichwohl verurteilt. Und in der Tat konnte sie nicht mit der gesetzlichen Strafe belegt werden, weil man ihr trotz unermüdlicher Verhörtätigkeit über die ihr ohnehin nachgewiesenen Umstände hinaus keinerlei Geständnis entlocken konnte. Nicht zuletzt dies ist es, was sie für den Neuen Pitaval zur Rätselfigur macht. Mit einer überlegenen Intelligenz, einer weitläufigen Bildung und einer großen Selbstbeherrschung ausgestattet, schien sie den Richtern »in den Gesetzen so bewandert", als sei das "preußische Landrecht [...] ihre Lieblingslectüre«. Unzufrieden mit der Defensionsschrift ihres Verteidigers,

21 Vgl. Michael Niehaus: Das Verhör. Geschichte - Theorie - Fiktion, München 2003, insbes. S. $285 \mathrm{ff}$. So heißt es etwa gegen Ende der Bekenntnisse: »wenn die Justiz mich zur Rechenschaft zieht, werd' ich keinen Augenblick mehr selbst entstehen; ich habe allzu lange über mich nachgedacht, als dass mich irgend eine Schwäche besiegen sollte. Man kann mich verdammen, man kann mich sogar vernichten; aber man kann mich nicht überführen und bestrafen." (BG 219) Die Giftmischerin wird, mit anderen Worten, als jemand imaginiert, den man im Verhör nicht zur Mitarbeit, zu einem rückhaltlosen Bekenntnis wird bewegen können. Im Gegenzug wären die rückhaltlosen Bekenntnisse einer Giftmischerin selbst als Teil des Kampfes anzusehen. 
hatte sie sich eine Verteidigungsstrategie zurechtgelegt, die sie sogar als eigenständige Schrift publizieren ließ. ${ }^{22}$

Die Strategie von Charlotte Ursinus bestand - grob gesagt - darin, die früheren Morde abzustreiten, sich selbst als suizidgefährdet darzustellen und für die Vergiftungen ihres Bediensteten temporäre Unzurechnungsfähigkeit geltend zu machen. Scharfsinnig macht sie sich dabei den problematischen Schluss zu eigen, dass die Unzurechnungsfähigkeit zum Zeitpunkt der Begehung einer Tat aus ihrer Unverständlichkeit abgeleitet werden kann. ${ }^{23}$

Insofern sie gleichwohl zu lebenslänglicher Festungshaft verurteilt worden ist, hat Charlotte Ursinus mit dieser Strategie nur begrenzt Erfolg gehabt. Es ist ihr auf diese Weise aber gelungen, ihre Subjektposition zu behaupten. Bis zu ihrem Tode 35 Jahre später ist sie eine Frau geblieben, die nur im Zustand zeitweiliger geistiger Zerrüttung zum Gift gegriffen und ansonsten ein untadeliges Leben geführt hat. Als vermögende Frau hat sie auf der Festung Glatz eine Vorzugsbehandlung genossen, eine Gesellschafterin gehabt, Besuche empfangen und vielfach wohltätig gewirkt. Am Ende wurde sie begnadigt. Sie durfte sich ihren Wohnsitz in der Stadt nehmen und verkehrte auch wieder in den vornehmen Kreisen. Schließlich soll sie, wie der Neue Pitaval zähneknirschend und fasziniert feststellt, "als eine Heilige gestorben sein« (NP 216). ${ }^{24}$

III.

Der Fall der Gesche Gottfried ist ungleich spektakulärer und verzweigter und kann hier nur in groben Umrissen skizziert werden. Das Hauptaugen-

22 Authentische Vertheidigung der verwittweten Geh.Räthin Ursinus, von ihr selbst aufgesetzt, Berlin/Leipzig 1804. Die Verteidigungsschrift ist im Übrigen auch - bezeichnenderweise - in der Darstellung des Neuen Pitaval vollständig abgedruckt (vgl. NP 201-211). Nebenher spricht sie dort auch mit Blick auf die Bekenntnisse einer Giftmischerin von "unmenschlichen Schriftstellern", die sie "als ein Ungeheuer anderen zum schrecklichen Beispiel aufgestellt« hätten (ebd., S. 10).

23 Originalton: Wenn "Befangenheit der Urtheilskraft, und Krankheit des Seelenorgans, die einzigen Bedingungen der Möglichkeit sind, eine Handlung zu begehen, da können auch sie allein nur hinreichende Gründe seyn, die Wirklichkeit der Handlung zu begreifen, und die wahre Entschuldigung solcher Vergehen ist die wahre Geschichte ihrer Entstehung." (Ebd., S. 13)

24 Für weitere Informationen zum Leben der Vorzugsgefangenen Charlotte Ursinus auf der Festung Glatz vgl. ausführlich Joachim Berke: Geschichten aus dem alten Schlesien, Norderstedt 2009, S. 53-105. 
merk soll dann wiederum auf jene Phase gerichtet werden, in der die Enttarnung stattgefunden hat, das Urteil aber noch aussteht.

Die 1885 geborene Gesche Gottfried wuchs in Bremen in geordneten kleinbürgerlichen Verhältnissen auf. An ihrem 21. Geburtstag heiratete sie Johann Miltenberg, den Sohn eines wohlhabenden Sattlermeisters aus der Nachbarschaft. 1813 vergiftet sie - wie mutmaßlich Charlotte Ursinus - als erstes ihren gesundheitlich zerrütteten Mann mit Arsenik, anderthalb Jahre später in kurzem zeitlichen Abstand ihre drei Kinder und ihre Eltern, dann ein halbes Jahr später auch ihren aus den napoleonischen Kriegen heimgekehrten Zwillingsbruder. In dieser Zeit unterhält Gesche eine Liebesbeziehung zu Christoph Michael Gottfried, einem engen Freund ihres verstorbenen Mannes. Nachdem sie ihre Familie ausgelöscht hat, glaubt sie, dass einer Heirat mit Gottfried nichts mehr im Wege steht. Als sie das Zögern ihres Geliebten bemerkt, verabreicht sie ihm - inzwischen schwanger - ebenfalls Gift und bewegt ihn in todkrankem Zustand zum Vollzug der Trauung. Drei Tage später stirbt er.

Um die wirtschaftliche Situation der nunmehrigen Gesche Gottfried ist es in den folgenden Jahren nicht gut bestellt, teils wegen eigener unnützer Geldausgaben - sie galt als eitel und mildtätig -, teils weil ihr erster Ehemann das väterliche Erbe verprasst und ihr zweiter Ehemann sich als wenig vermögend erwiesen hat. Jedenfalls verstrickt sie sich zunehmend in ein Netz aus Schulden, das wohl auch zum auslösenden Moment der zweiten Vergiftungsserie sechs Jahre später wird. Zwischen 1823 und 1827 tötet sie noch einmal insgesamt sieben Personen mit Gift. Hatte sich die erste Vergiftungsserie gegen die Familie gerichtet, fallen ihr nun Menschen aus der persönlichen Umgebung zum Opfer, darunter ein Verlobter, ihre treue Magd samt ihrem Kleinkind, ihre beste Freundin, ein väterlicher Schuldner. Neben den tödlichen werden ihr später auch zahlreiche nichttödliche Vergiftungen nachgewiesen. Schließlich wird sie durch den in ihrem Hause wohnenden Radmachermeister Rumpff enttarnt, der, nachdem er monatelang an rätselhaften Krankheitssymptomen gelitten hat, einen vergifteten Schinken zur Apotheke trägt. ${ }^{25}$

Auch hier führt also erst der manifest gewordene Vergiftungsversuch zum Ermittlungsverfahren, und die Falldarstellung im Neuen Pitaval beginnt entsprechend mit der Enttarnung der Gesche Gottfried im Jahre 1828. Anders aber

25 Peer Meter hat sich in verschiedenen Veröffentlichungen um den Fall Gesche Gottfried bemüht; vgl. vor allem Peer Meter: Gesche Gottfried. Eine Bremer Tragödie, Bremen 2010 . 
als bei Charlotte Ursinus gibt es Gerüchte um Gesche Gottfried auch schon vorher. Wie kann es auch anders sein, wenn in kurzer Zeit ihre gesamte Familie dahingerafft wird? Bereits 1815 waren nach dem Tod ihres letzten Kindes Stimmen laut geworden, die zu einer Exhumierung und Sektion der Leiche geführt hatten. Aber der sezierende Arzt hatte damals versichert, "[d] er Knabe sei an einer Verschlingung der Eingeweide gestorben" (NP 308). Die Unnachweisbarkeit des Giftes, gepaart mit medizinischer Uninformiertheit, hatte damals den Verdacht zum Schweigen gebracht.

Oder besser: Das Gerücht hatte sich auf eine andere Ebene verlagert, die für das Verständnis dieser Tötungsart und die Subjektposition des Täters bzw. der Täterin von großer Bedeutung ist: Eine Frau, die in ihrer Umgebung so viele Tote zu beklagen hat, muss eine vom Schicksal Verfolgte sein. Das Haus der Gesche Gottfried galt als ein Unglückshaus, und der Neue Pitaval erläutert: "so auffällig war das ungewöhnliche Unglück dieser Frau geworden, daß ein hochberühmter Kanzelredner in Bremen [...] auf der Kanzel für die >christlich starke Dulderin öffentliche Fürbitten hielt.« (NP 257)

Während Gesche Gottfried im Verborgenen für ihre Opfer das Schicksal war, erschien sie in der Öffentlichkeit selbst als vom Schicksal Verfolgte. Sie hat diese Subjektposition wohl zu stützen gewusst, indem sie sich bei neuen Todesfällen etwa auf die »dunkeln Wege des Schicksals" berief, »das doch immer unser Bestes will« (NP 340) usw. Und in ihrer Anfangszeit hat sie sogar die bei einer Wahrsagerin über sie verhängte Prophezeiung, ihre Familie werde sterben, sie selbst aber am Leben bleiben, unters Volk gebracht. ${ }^{26}$ Auf diese Weise ist es ihr gelungen, die eigenen Taten zur Tarnung ihrer Aufdeckung zu verwenden. Das geht nicht ohne die Komplizenschaft der Gesellschaft, die bereit ist, die Augen zu verschließen, und an das Schicksal - oder an die Vorsehung und ihre Werkzeuge - zu glauben. ${ }^{27}$

In gewissem Sinne könnte man sagen: Die Personen in dem sozialen Umfeld, in dem Gesche Gottfried sich bewegte und sich anerkannt fühlen durfte als aufopferungsbereite und mildtätige Dame, befanden sich in der Position, Unwissenheit vorschützen zu können. Mit der Verhaftung, die ja zunächst nur einen Vergiftungsversuch betraf, wurde gewissermaßen ein »Vorhang« weggezogen. ${ }^{28}$ Noch bevor die Ermittlung in Gestalt von Verhören Re-

${ }^{26}$ Vgl. den Abschnitt »Vom Kartenlegen und Prophezeien«, in: Peer Meter: Gesche Gottfried, S. 135-137.

27 Vgl. genauer Michael Niehaus: Schicksal sein, S. 147f. Der Komplex des Wahrsagens und des Giftmordes sind auf einer strukturellen Ebene miteinander verknüpft, weil die Giftmischerin und der Giftmischer mittels ihrer Taten Prophezeiungen wahr machen können.

28 Peer Meter: Gesche Gottfried, S. 55. 
sultate zeitigen konnte, hatte die Öffentlichkeit die Geschichte der Gesche Gottfried neu zusammengesetzt. Sie hatte ihre Morde zusammengezählt, und so konnten manche Personen aus ihrer Umgebung ihre eigenen unerklärlichen Krankheitssymptome nun einer Ursache zuschreiben - teils zu Recht, teils zu Unrecht. Das `Unglaublicher, das sich bei Charlotte Ursinus nicht bestätigt hat, bewahrheitete sich hier.

Der Neue Pitaval setzt in seiner Falldarstellung den noch darüber hinausgehenden "Heißhunger« des Volkes "nach dem Phantastischen" - es ging das Gerücht, die Gottfried habe ihre Kinder totgekitzelt, um das berüchtigte Aqua Toffana zu bekommen; sie habe das Fleisch ihrer Kinder als Speise zubereitet, sei schon von ihrer Mutter in die Giftmischerei eingewiesen worden usw. (vgl. NP 263f.) - gegen den "psychologische[n]« Schlüssel zur Erklärung dieser Figur, welcher »erst nach unsäglicher Mühe und nach Jahren von wissenschaftlichen Männern gefunden« (NP 264) worden sei. ${ }^{29}$ Damit ist der Verteidiger Gesche Gottfrieds, Friedrich Leopold Voget, gemeint, der zur Erhellung dieses psychologischen Rätsels noch vor der Verurteilung ein umfängliches Buch mit ihrer Lebensgeschichte veröffentlichte, dem er nach der Hinrichtung 1831 ein zweites Buch mit dem Prozesshergang und dem Betragen der Inquisitin in der Haftzeit folgen ließ. ${ }^{30}$

Auch dieses Buch zeigt: Die Spanne zwischen der Enttarnung ${ }^{31}$ und der Verurteilung ist eine Zeit der Phantasmen auf der einen und des Durch-

${ }^{29}$ Diesem Auffinden eines "Schlüssels« zum Trotz wird etwas weiter unten darauf insistiert, dass Gesche Gottfried ein nicht restlos entschlüsselbares Rätsel ist: „Der äußere Mensch, die Gottfried, wie sie straffällig vor dem Gesetz erscheint, ist darin [in den Akten, M.N.] vielleicht splitternackt dargestellt. Aber durch kein articulirtes Verhör und durch keine Protokolle, die ein Richter führt, kann eine Erscheinung, wie die ihre, psychologisch ergründet werden. Um die feinern Fäden zu verfolgen, wie aus der menschlichen Natur ein solches entmenschtes Wesen werden konnte, sind die Federn der Gerichtsstube, das Actenpapier zu grob.« (NP 266).

30 Friedrich Leopold Voget: Lebensgeschichte der Giftmörderin Gesche Margarethe Gottfried. In gekürzter Fassung herausgegeben und mit einem Nachwort versehen von Eckart Oehlenschläger, Bremen 1976. Die Kürzungen bei dieser Neuedition beziehen sich lediglich auf den zweiten Teil, in dem verschiedene Prozessualia weggelassen worden sind. Peer Meter beurteilt die Rolle von Voget sehr kritisch; insbesondere bestreitet er, dass Voget »bei der Übernahme der Verteidigung nur ein menschliches Bemühen um diese Frau im Sinn gehabt« hätte; vielmehr habe er schon zu diesem Zeitpunkt den Plan einer Buchpublikation gehegt (Peer Meter: Gesche Gottfried, S. 188).

31 Der für das Verständnis des Verhältnisses von Tötungsart und Ermittlungspraxis entscheidende Vorgang der Enttarnung wird im Falle der Gesche Gottfried noch einmal verdoppelt bzw. emblematisch überhöht. Als die Wärterfrauen im Gefängnis die enttarnte Gesche Gottfried im Stadthause »dem Regiment zufolge, entkleiden mussten", fanden sie »dreizehn Corsette, eins über dem andern [...]. Ihre lieblichen rothen Wangen waren Schminke, und nachdem alle Toilettenkünste entfernt, stand an der Stelle der blühenden, 
und Aufarbeitens im Ermittlungsverfahren auf der anderen Seite. Die langwierige Ermittlungstätigkeit im Falle Gesche Gottfried besteht in endlosen Verhören: Jede mögliche Vergiftung der letzten beiden Jahrzehnte muss zum Thema gemacht und gegebenenfalls zum Geständnis gebracht werden. ${ }^{32}$ Gesche Gottfried gibt in diesen Verhören keine so 'gute Figur ab wie Charlotte Ursinus. Sie wird endgültig sdemontiert. In anderer Weise als die historische Charlotte Ursinus und die anonyme Giftmischerin in den Bekenntnissen einer Giftmischerin ist aber auch Gesche Gottfried jenseits der Reuer. Oder genauer: Es wird ihr attestiert, zu einer wahrhaftigen Reue, zu einem rückhaltlosen Geständnis nicht aus Stärke, sondern aus Schwäche nicht in der Lage zu sein: Im Neuen Pitaval wird unerbittlich erklärt:

Sie bekannte, aber nicht gestachtelt von Gewissensunruhe, nicht gerührt durch den Zorn Gottes, der ihr Herz zerschmettert, sie bekannte, weil sie nicht mehr die Kraft hatte, die Lüge zu halten. Nicht mit einem Male, es war ein fortgesetztes zweijähriges Bekennen, und auch dieses Bekennen war ein fortgesetztes neues Lügengewebe [...]. (NP 265)

Voget gibt - wie im Neuen Pitaval zusammengefasst wird - in seinem Buch von seiner Mandantin das »vollständige[ ] Bild« eines »hohlen Wesens, eigentlich nur eines Schemens«. Gesche Gottfried erscheint als eine vollendete "Heuchlerin", bei deren "proteischen Windungen" man "jede Gemüthsregung benutzen musste«, »um ihrer schnell vorübergehenden Zerknirschung ein Geständniß abzupressen« (NP 267).

Gesche Gottfried kann als Paradigma eines Subjekts aufgefasst werden, das, scheinbar in unserer Mitte, aus allen sozialen Bindungen herausgefallen ist. Aber das liegt nicht daran, dass sie aus einem anderen Holze geschnitzt, aus einem anderen Stoff gemacht ist. Sie wird trotz ihrer unglaublichen Taten - und letztlich gerade wegen ihrer Schwäche, ihrer Unfähigkeit zum reuevollen Geständnis - als »ein menschliches Wesen, gleich uns« (NP 267) vorgestellt. Sie selbst hat sich in den Augen der >Wissenschaft $<$ auf die "Sündenbahn« (NP 351) begeben, die ins Jenseits des Sozialen geführt hat. ${ }^{33}$

wohlbeleibten Dame vor den erschreckten Weibern ein blasses, angstvoll verzerrtes Gerippe." (NP 265) Für das Protokoll der Entkleidung vgl. Peer Meter: Gesche Gottfried, S. 53.

32 Bei Meter wird der Gang der Untersuchung bzw. der Ablauf der Verhöre detailliert dargestellt (vgl. ebd., S. 51-176); möglich war dies, weil die 1950 in Moskau wiederaufgefundenen Strafprozessakten des Inquisitionsverfahrens gegen Gesche Gottfried 1987 von der DDR an die Stadt Bremen zurückgegeben wurden.

33 Voget hat sich in seinem Vorwort nichts Geringeres zum Ziel gesetzt als »die Entschleierung des Innern eines menschlichen Wesens, welches mit tausend anderen von einem 
Sie ist ein verantwortliches Monster, ${ }^{34}$ dem man die Schuld geben muss. Den "Trieb zum Vergiften" - »nicht finstere Mächte, böse Dämonen, sie selbst impfte ihn sich ein« (NP 351).

Wenn man das Wissenschaft nennen will (und warum sollte man es nicht), so wird Gesche Gottfried das Dämonische also durch die Psychologie ausgetrieben. Aber dies geschieht auf eine dämonische Art und Weise. Auch wurde Gesche Gottfried in ihrer Haftzeit wiederholt von Angstvisionen und Träumen heimgesucht. Insbesondere erschienen ihr ihre Opfer. ${ }^{35}$ Der Neue Pitaval hat hierfür die naheliegende dämonologische bzw. moralisch-okkulte Interpretation parat, dass sich das »Unsichtbare [...] für die Verleugnung durch furchtbare Visionen" rächte, »die die Verbrecherin gespensterhaft bei Nacht und bei Tage ängstigten." (NP 348)

Von all dem erfuhr die Öffentlichkeit zwischen 1828 und 1831 allenfalls gerüchteweise, da das Ermittlungsverfahren unter Ausschluss der Öffentlichkeit stattfand. Auch im Falle Gesche Gottfrieds wurde aus diesem Mangel Literatur geboren, in der - wenn auch in ganz anderer Weise als in den Bekenntnissen einer Giftmischerin - das Dämonische zu seinem Recht kam. Unter dem Pseudonym Weissenburg der Ältere erschien 1829 die Buchform eines nie aufgeführten Melodrams mit dem Titel Gesinia, die Teufelsbraut. ${ }^{36}$ Die Informationssperre bei den Ermittlungen gebiert Ungeheuer. Sie erleichtert

und demselben Anfangspunkt beginnend, auch äußerlich mit tausend eine gleiche Bahn weitergehend, vor unsern Augen zu der furchtbarsten Verruchtheit herabsinkt - jedoch so, dass wir den Keim zu gleichem oder ähnlichem Verfall in unserem eigenen Herzen wahrnehmen" (ebd., S. 7). Vogets Kriminalpsychologie ist also bis zu einem gewissen Grade moralistisch. Man mag dies als eine Erbschaft des 18. Jahrhunderts deklarieren, bringt sich dadurch aber um die Einsicht, dass nur die moralische Dimension die Kriminalpsychologie davor bewahrt, das begutachtete Subjekt aus der menschlichen Gemeinschaft auszuschließen.

34 Vgl. Michael Niehaus: Das verantwortliche Monster, in: Monströse Ordnungen. Zur Typologie und Ästhetik des Anormalen, hg. von Achim Geisenhanslüke und Georg Mein, Bielefeld 2009, S. 81-102.

35 Vgl. Peer Meter: Gesche Gottfried, S. 70f.

36 Das Melodram ist natürlich nicht die einzige und nicht die erste Publikation, die während der Ermittlung zu Gesche Gottfried erschien. U.a. kursierten verschiedene Flugschriften (vgl. ebd., S. 178ff.). Ebenfalls zuvor war ein Büchlein mit dem Titel Geschichte der berüchtigten Bremer Giftmischerin Margarethe Gesina Gottfried geb. Timme, und ausfïhrliche Erä̈hlung ihrer schauerlichen Unthaten. Nebst einem Anhange, welcher ihre gerichtlichen Aussagen enthält, Nach authentischen Quellen bearbeitet erschienen, dessen Verfasser kurioserweise mit demselben Pseudonym auftrat: Weißenburg der Ältere (es sind dies die beiden einzigen Publikationen, die unter diesem Pseudonym verzeichnet sind). Der Richter, der die Untersuchung des Falles leitete, sah sich auf diese Publikation hin genötigt, in der Zeitung bekannt zu machen, dass es sich bei diesem Machwerk um eine pure Fälschung handle, um fiktive Dokumentarliteratur gewissermaßen, und um eine Lüge mit kurzen Beinen. 
die Konstruktion der Subjektposition der Giftmischerin ohne Rücksicht auf das Realitätsprinzip. ${ }^{37}$

Im Melodram wird das Dämonische in Reinform präsentiert. Es redet Klartext: Gesche Gottfried lässt sich mit dem Teufel ein. Vorgeführt wird eine unhaltbare Subjektposition. Am Ende, nach ihrer Überführung, möchte die Teufelsbraut ihr unbedacht abgelegtes Geständnis widerrufen. "Ich muß die Richter durch verschiedene Aussagen zu täuschen suchen «. ${ }^{38}$ Auf dieser schändlichen Absichtsbekundung kommt das Moralisch-Okkulte ${ }^{39}$ unmittelbar zur Geltung. Es ertönt ein »starker Donnerschlag« und »mehrere Blitze" schlagen ein. Der Kerker steht in Flammen und der Teufel erscheint. Die Bühne wandelt sich zur Hölle. Am Ende wird Gesinia »in die Schlucht des Hintergrundes" geschleudert, und der Schauplatz vernichtet sich selbst - »die Felsen wanken und stürzen unter Geprassel zusammen, ein starker Feuerregen fällt, die Glocke schlägt Eins und der Vorhang fällt«. ${ }^{40}$

Am Ende soll jedoch ein Stück Literatur stehen, in dem der Teufel die Gesche Gottfried nicht holt. 1828 - also noch vor dem Melodram - inspirierte der Fall Gesche Gottfried Adelbert von Chamisso zu einem Die Giftmischerin betitelten Rollengedicht. Das Ende der Giftmischerin wird auch hier vorweggenommen: In ihrer Rede auf dem Richtplatz, adressiert an den Henker und uns alle, mutet Chamisso Gesche Gottfried - ohne ihren Namen zu nennen - das reuelose Insistieren auf der unmöglichen Subjektposition zu, die in der Figur der Giftmischerin impliziert ist. In der ersten Strophe erklärt sie, ihre »Leichenrede selber halten« zu wollen:

Was schauet ihr mich an so grausenvoll?

Ich führte Krieg, wie jeder thut und soll,

Gen feindliche Gewalten.

Ich that nur eben, was ihr alle thut,

Nur besser; drum, begehret ihr mein Blut,

So thut ihr gut.

37 Vgl. zu diesem Melodram ausführlich Michael Niehaus: Der Fall im Melodram: Gesinia, die Teufelsbraut, in: Das Melodram, ein Medienbastard, hg. von Bettine Menke, Armin Schäfer und Daniel Eschkötter, Berlin 2013, S. 190-203.

38 [Weißenburg der Ältere]: Gesinia, die Teufelsbraut. Oder der 6te März. Melodrama in 3 Perioden, jede in 2 Abtheilungen, o.O. 1929, S. 105.

39 Der Begriff des "Moralisch-Okkulten" wurde insbesondere von Peter Brooks verwendet, um das Eigentümliche der melodramatischen Imagination zu beschreiben; vgl. Peter Brooks: Die melodramatische Imagination, in: Und immer wieder geht die Sonne auf. Texte zum Melodramatischen im Film, hg. von Christian Cargnelli und Michael Palm, Wien 1994, S. 35-64.

40 [Weißenburg der Ältere]: Gesinia, die Teufelsbraut, S. 108. 
Und entsprechend endet dieses bestürzende Gedicht:

Ich habe mich zu sicher nur geglaubt,

Und büß' es billig mit dem eig'nen Haupt,

Daß ich der Vorsicht einmal mich begeben.

Den Fehl, den einen Fehl bereu' ich nur,

Und gäbe, zu vertilgen dessen Spur,

Wie viele eurer Leben!

Du, schlachte mich nun ab, es muß ja sein.

Ich blicke starr und fest vom Rabenstein

Ins Nichts hinein. ${ }^{41}$

41 Adelbert von Chamisso: Die Giftmischerin, in: ders., Sämtliche Werke in vier Bänden, Bd. 1, hg. von Adolf Bartels, Leipzig [1926], S. 185-187. 